EXTENDED REPORT

\title{
Lumbosacral radiographic signs in patients with previous enteroarthritis or uroarthritis
}

\author{
A Mannoja, J Pekkola, M Hämäläinen, M Leirisalo-Repo, L Laasonen, L Kivisaari
}

Ann Rheum Dis 2005;64:936-939. doi: 10.1136/ard.2004.027086

See end of article for authors' affiliations

Correspondence to: Dr Antti Mannoja, Marjatie 6b, FIN-00780, Helsinki, Finland; antti. mannoja@helsinki.fi

Accepted

9 November 2004

Published Online First

11 November 2004

\begin{abstract}
Objective: To investigate if there are differences in radiological findings in lumbosacral spine radiographs between different subgroups of patients with previous reactive arthritis.

Methods: 95 patients with previous enteroarthritis $(n=53)$, uroarthritis $(n=37)$, or Reiter's syndrome, aetiology unknown $(n=5)$ were included in the study. Lumbosacral radiographs were taken 7 to 38 years after the initial arthritis. Three independent observers read the radiographs. Spinal changes included squaring of vertebrae, Romanus lesions, syndesmophytes, and osteophytes. Sacroiliitis was recorded according to the New York and Stoke methods. Signs of enthesitis in the iliac crest and disc space narrowing were recorded. Interobserver reliability and intraobserver reproducibility were determined.

Results: $23 \%$ of patients had grade 2-4 sacroiliitis (New York criteria) and $14 \%$ had syndesmophytes. There was more frequent sacroiliitis $(32 \% \vee 13 \%)$ in uroarthritis than in enteroarthritis, and more syndesmophytes (mean 0.54 v 0.15 per patient; prevalence $24 \%$ v $6 \%$, respectively). In other radiological features, no significant differences were present between the groups. In the material as a whole, patients with sacroiliitis of grade 2 or more had significantly less disc space narrowing both in patient comparisons $\left(\chi^{2}\right.$ test) and in numbers of spaces involved (Mann-Whitney test). Interobserver and intraobserver agreement, calculated using Cohen's $\kappa$ method, varied from 0.2 to 1 .

Conclusions: Syndesmophytes and sacroiliitis are more common in patients with previous uroarthritis than in those with previous enteroarthritis, but radiological findings in lumbosacral spine radiographs are characteristically similar.
\end{abstract}

$\mathrm{R}$ eactive arthritis is a subgroup of the spondylarthropathies, other members of which are ankylosing spondylitis, arthritis associated with inflammatory bowel disease, psoriatic arthritis, and undifferentiated spondylarthropathy. Reactive arthritis is described as a sterile arthritis occurring after a primary infection remote from the joint at another site such as the gastrointestinal or urogenital tract. ${ }^{2}$ Several criteria for diagnosing reactive arthritis have been proposed, but at present there is no general agreement on their use..$^{3-5}$

Reactive arthritis is classically divided into uroarthritis and enteroarthritis, following urogenital and enteral infection, respectively. The most common pathogens underlying enteroarthritis are yersinia, salmonella, campylobacter, and shigella species, ${ }^{6}$ and for uroarthritis Chlamydia trachomatis. ${ }^{7}$

Spondylarthropathies differ in the radiological appearances in the spine. In 1974, McEwen et $a l^{8}$ made a distinction in the radiological characteristics of the spine between so called category 1 (ankylosing spondylitis and spondylarthropathy associated with inflammatory bowel disease) and category 2 (Reiter's disease and psoriatic arthritis). Later on, Helliwell et $a l^{9}$ confirmed some but not all of these differences. They confirmed the asymmetry of syndesmophytes, the less severe changes, and the distinctive form of the syndesmophytes in psoriatic arthritis compared with ankylosing spondylitis and spondylarthropathy associated with inflammatory bowel disease. However, the reactive arthritis subgroup in this later study was so small that definitive conclusions about this form of arthritis in relation to other subgroups could not be drawn. The most commonly affected segments of the spine in Reiter's disease are the lower dorsal and upper lumbar regions. ${ }^{8}{ }^{10}$

Radiography is still the standard for diagnosing chronic sacroiliitis. ${ }^{11}$ Radiological signs of spondylarthropathy (syndesmophytes or sacroiliitis) are observed in $10-30 \%$ of patients with previous reactive arthritis. ${ }^{12}{ }^{13}$ Our aim in this study was to determine whether the proportions of types of syndesmophytes and the characteristics of other spondylarthropathy related signs, such as sacroiliitis, differ between uroarthritis and enteroarthritis in lumbosacral radiographs of patients with previous definite reactive arthritis.

\section{METHODS \\ Patients}

The material of this study included radiographs of 95 Finnish patients with diagnosis of reactive arthritis or Reiter's syndrome. Patients were treated in the Department of Medicine at Helsinki University Central Hospital. The acute phase of reactive arthritis or Reiter's syndrome began in 1955 to 1986. A follow up study with lumbosacral radiographs was carried out in 1991 to 1995. The average age of the patients at the time of radiography was 48.7 years (range 30 to 86 ). There were 60 men and 35 women in the study. The patients were grouped into one of three categories: enteroarthritis, uroarthritis, or Reiter's syndrome of unknown aetiology. The enteroarthritis group $(n=53)$ consisted of 46 patients with yersinia, five with salmonella, and two with enteritis of unknown aetiology as the triggering infection. The uroarthritis group $(\mathrm{n}=37)$ consisted of 13 patients with Chlamydia trachomatis, 16 with non-gonococcal urethritis, six with gonococcal urethritis, and two with prostatitis of unknown aetiology as the triggering infection or focus. Five patients who had complete or incomplete Reiter's syndrome but no known preceding infection formed the aetiology unknown Reiter's syndrome group. HLA-B27 status was available for 89 patients.

All patients had a typical clinical picture of reactive arthritis. The diagnosis of Chlamydia trachomatis was based on urethral/cervical culture or serology, or both, by immunofluorescence; diagnosis of gonococcus was based on urethral/ cervical culture; and diagnosis of yersinia and salmonella was based on stool culture or serology, or both. 
Table 1 Characteristics of the patients

\begin{tabular}{|c|c|c|c|c|}
\hline & Enteroarthritis & Uroarthritis & $\begin{array}{l}\text { Reiter's syndrome, aetiology } \\
\text { unknown }\end{array}$ & Total \\
\hline $\mathrm{n}$ & 53 & 37 & 5 & 95 \\
\hline Male/female & $21 / 32$ & $34 / 3$ & $5 / 0$ & $60 / 35$ \\
\hline Age at follow up (years) (mean (range)) & 49.5 (30 to 86$)$ & 47.5 (33 to 63 ) & 48.2 (34 to 61$)$ & 48.7 (30 to 86$)$ \\
\hline Duration of follow up (years) (mean (range)) & $18.4(7$ to 24$)$ & $19.2(14$ to 34$)$ & 21.1 (14 to 38$)$ & 18.9 (7 to 38$)$ \\
\hline 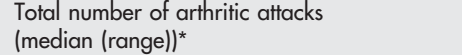 & $1(1$ to 6$)$ & $1(1$ to 10$)$ & $2(1$ to 3$)$ & $1(1$ to 10$)$ \\
\hline HLA-B27+ & $40 / 50(80 \%)$ & $30 / 36(83 \%)$ & $2 / 3(67 \%)$ & $72 / 89(81 \%)$ \\
\hline
\end{tabular}

For patients with negative cultures and serology, a history of preceding symptomatic urological infection or gastroenteritis was applied in classifying patients into the enteroarthritis or uroarthritis groups. Patients had no clinical or microbiological evidence of purulent arthritis, and we excluded other rheumatic diseases according to routine clinical practice. The general characteristics of the groups are described in table 1. The study is a part of the long term outcome study of reactive arthritis. The study protocol was approved by the ethics committee of the Helsinki University Central Hospital.

\section{METHODS}

Conventional lumbosacral plain radiography with anteroposterior and lateral projections was used. The lumbosacral radiographs were read by three independent observers in 2002. Observers did not know the reactive arthritis subgroup or the clinical features of the patients. Two of the observers were radiologists ( $\mathrm{MH}$ and $\mathrm{JP}$ ) and one an orientated general practitioner (AM). A structured form was used in interpreting the radiographs. Before reading the radiographs, observers participated in a common session where typical findings were discussed and the use of the form was practised with an experienced radiologist (LL). A preliminary reading of the material from 10 patients was carried out, followed by a practical session with advice on filling out the form. After first reading the entire material, radiographs of 20 randomly selected patients were read again in 2003 by the same three observers blinded to name and day of birth of patients (year of birth was available), to assess intraobserver reproducibility.

Spinal changes were assessed from T12 to S1. Squaring of vertebrae and Romanus lesions were interpreted with regard to vertebrae, and, in the case of Romanus lesions, also the upper anterior and lower anterior corners of the vertebrae. In later analysis, these data were converted to either the presence or the absence of these signs in a patient.
Marginal and non-marginal syndesmophytes and osteophytes were recorded with regard to every intervertebral space so that location could be anterior, right lateral, left lateral, or posterior. For any given location no more than one of these three options (non-marginal/marginal syndesmophytes and osteophytes) could be recorded. Syndesmophytes were graded from 1 to 3 . Grade 1 was used when a syndesmophyte did not reach over the intervertebral border of a vertebra; grade 2 when a syndesmophyte reached over the border but was not bridging; and grade 3 when there was a bridging syndesmophyte. Osteophytes were not graded. In the analysis, the grade of a syndesmophyte was determined as a rounded average of grades of the observers. Syndesmophytes were considered as bilaterally asymmetrical when a difference was found in the type or grade of lateral syndesmophyte at the same level. The symmetry of anterior and posterior syndesmophytes was not determined. In the analysis a radiological sign was considered to be present when at least two of the observers noticed it.

Sacroiliitis was assessed using both the modified New York and the Stoke methods. ${ }^{14}{ }^{15}$ In case of disagreement between the three readers, a majority decision was reached. In further analysis, sacroiliitis was grouped either as grades $0-1$ and 24 , or as grades $0-2$ and 3-4.

Iliac enthesitis was looked for separately on the left and right sides. Disc space narrowing was determined at every intervertebral space. Interobserver and intraobserver agreement on non-marginal syndesmophytes, marginal syndesmophytes, osteophytes, disc space narrowing, and iliac enthesitis was calculated with respect to every recorded location on the spine.

\section{Statistics}

Statistical differences between the groups were calculated pairwise using the Pearson $\chi^{2}$ method (and Fisher's exact test

Table 2 Spinal radiographic signs in the groups

\begin{tabular}{|c|c|c|c|c|}
\hline & Enteroarthritis $(n=53)$ & Uroarthritis $(n=37$ ) & $\begin{array}{l}\text { Reiter's syndrome, } \\
\text { aetiology unknown } \\
(\mathrm{n}=5)\end{array}$ & Total (95) \\
\hline Squaring of vertebrae, presence (\%) & $5(9)$ & $0(0)$ & $0(0)$ & $5(5)$ \\
\hline Romanus lesions, presence (\%) & $1(2)$ & $0(0)$ & $0(0)$ & $1(1)$ \\
\hline Syndesmophytes, presence (\%) & $3(6)^{*}$ & $9(24)^{*}$ & $1(20)$ & $13(14)$ \\
\hline Non-marginal/marginal, presence & $2 / 1$ & $7 / 2$ & $0 / 1$ & $9 / 4$ \\
\hline Syndesmophytes, mean number per patient (range) & $0.15^{*}(0$ to 3$)$ & $0.54^{*}(0$ to 6$)$ & $0.40(0$ to 2$)$ & $0.32(0$ to 6$)$ \\
\hline Non-marginal/marginal syndesmophytes (n (\%) non-marginal) & $5 / 3(63)$ & $17 / 3(85)$ & $1 / 1(50)$ & $23 / 7(77)$ \\
\hline Anterior syndesmophytes (n (\%)) & $4(50)$ & $9(45)$ & $0(0)$ & $13(43)$ \\
\hline Unilateral syndesmophytes, number (\%) & $3(38)$ & 7 (35) & $1(50)$ & 11 (37) \\
\hline Bilateral asymmetric syndesmophytes (n (\%)) & $0(0)$ & $2(10)$ & $1(50)$ & $3(10)$ \\
\hline $\begin{array}{l}\text { Mean number of syndesmophytes in patients with } \\
\text { syndesmophytes (range) }\end{array}$ & 2.7 (2 to 3 ) & $2.2(1$ to 6$)$ & $2(2)$ & $2.3(1$ to 6$)$ \\
\hline Mean grade of syndesmophytes in patients with & $2.7(2001$ & $2.2(100)$ & & 2.5111001 \\
\hline syndesmophytes (range) & $2.5(1$ to 3$)$ & $2.3(1$ to 3$)$ & $3.0(3)$ & 2.4 (1 to 3 ) \\
\hline Osteophytes, presence (\%) & $22(42)$ & $13(35)$ & $2(40)$ & $37(39)$ \\
\hline Disc space narrowing, presence $(\%)$ & $15(28)$ & $13(35)$ & $0(0)$ & $28(29)$ \\
\hline
\end{tabular}


Table 3 Sacroiliitis and iliac enthesitis in the groups

\begin{tabular}{|c|c|c|c|c|}
\hline & Enteroarthritis $(n=53$ ) & Uroarthritis $(n=37)$ & $\begin{array}{l}\text { Reiter's syndrome, aetiology } \\
\text { unknown }(n=5)\end{array}$ & Total $(n=95)$ \\
\hline Sacroiliitis (NY) grade 2-4 (\%) & $7(13)^{*}$ & $12(32)^{*}$ & $3(60)$ & $22(23)$ \\
\hline Sacroiliitis (Stoke) grade 2-4 (\%) & $8(15)$ & $11(30)$ & $3(60)$ & $22(23)$ \\
\hline Sacroiliitis (NY) grade 3-4 (\%) & $4(8)$ & $8(22)$ & $2(40)$ & $14(15)$ \\
\hline Sacroiliitis (Stoke) grade 3-4 (\%) & $4(8)$ & $6(16)$ & $3(60)$ & $13(14)$ \\
\hline Unilateral sacroiliitis (NY) grade $2-4(\%)$ & $1 / 7(14)$ & $4 / 12(33)$ & $0 / 3(0)$ & $5 / 22(23)$ \\
\hline Unilateral sacroiliitis (Stoke) grade 2-4 (\%) & $3 / 8(38)$ & $3 / 11(27)$ & $0 / 3(0)$ & $6 / 22(27)$ \\
\hline lliac enthesitis (\%) & $2(4)$ & $3(8)$ & $1(20)$ & $6(6)$ \\
\hline
\end{tabular}

for small groups when needed) or the Mann-Whitney U test. Interobserver reliability and intraobserver reproducibility were calculated using Cohen's $\kappa$ method. The cut off point for statistical significance was $\mathrm{p}<0.05$. In all calculations, SPSS version 11.0 statistical software was used.

\section{RESULTS}

No significant differences in the average age of patients during acute reactive arthritis, in the duration of follow up, in the total number of arthritic attacks, or in HLA-B27 positivity were found between the uroarthritis, enteroarthritis, and aetiology unknown Reiter's syndrome groups. The proportion of male patients in the uroarthritis and Reiter's syndrome groups was significantly higher than in the enteroarthritis group.

Syndesmophytes were more commonly present in the uroarthritis group (24\%) than in the enteroarthritis group $(6 \%, p=0.010)$. In the uroarthritis group, patients had more syndesmophytes than in the enteroarthritis group $(p=0.014)$.

There were no significant differences in marginality/nonmarginality, location (anterior, posterior, lateral), symmetry, or mean number of syndesmophytes in patients with syndesmophytes when comparing the groups (table 2). Also the average grade of syndesmophytes was fairly similar. There were no posterior syndesmophytes.

Sacroiliitis grades 2-4, using New York criteria, was more common in the uroarthritis group $(32 \%)$ than in the enteroarthritis group $(13 \%, p=0.028)$. When using Stoke criteria, the difference was not significant but was consistent with the results obtained with the New York criteria. When stricter criteria for sacroiliitis were used, there were no significant differences in frequency of grade 3-4 (table 3). No differences were present in unilaterality of sacroiliitis.

All the squaring of vertebrae and Romanus lesions were in the enteroarthritis group, but there were no significant differences between the groups. The frequency of iliac enthesitis did not differ between the groups (table 3 ).

The frequency and numbers (data not shown) of osteophytes and disc space narrowing were not significantly different between the main groups. In the material as a whole, patients with sacroiliitis by New York or Stoke grades
2-4 or 3-4 had less disc space narrowing than those with grades $0-1$ or $0-2(p<0.05)$, both in patient comparisons $\left(\chi^{2}\right.$ test) and in numbers of spaces involved (Mann-Whitney test). The mean (SD) age of patients with sacroiliitis grades 2-4 using the New York system (47.8 (7.1) years) was not significantly different from that of patients without sacroiliitis grades 2-4 (48.9 (9.2) years), neither were there any differences in mean duration of follow up between patients with sacroiliitis grades 2-4 (19.4 (5.0) years) and those without sacroiliitis grades 2-4 (18.6 (3.7) years). Analysis by dividing patients into sacroiliitis grades $0-2 \vee 3-4$ by New York criteria or applying the Stoke criteria did not change the results (data not shown). The results of radiographic findings are given in tables 2 and 3 .

When comparing the subgroups of the three main groups (yersinia, salmonella, chlamydia, gonococcus, non-gonococcal urethritis, prostatitis, enteroarthritis of unknown aetiology, and Reiter's syndrome of unknown aetiology), there were no significant differences with respect to sacroiliitis, syndesmophytes, iliac enthesitis, Romanus lesions, or squaring of the vertebrae (data not shown).

One of the patients in the gonococcal urethritis subgroup had serological evidence and one had culture based evidence of simultaneous chlamydial infection. If other gonococcal urethritis cases were omitted from the comparison between the uroarthritis and enteroarthritis groups, the statistical significance of the difference in the presence of syndesmophytes, grades 2-4 New York sacroiliitis, or the number of syndesmophytes did not change (data not shown).

Interobserver reliability and intraobserver reproducibility varied from 0.2 to 1 (table 4 ).

\section{DISCUSSION}

A mean of 19 years after acute reactive arthritis, $14 \%$ of patients had syndesmophytes in lumbosacral spine radiographs and $14-15 \%$ had grade 3-4 sacroiliitis. This study supports the view that the reactive arthritides are uniform in the character of the radiological findings but that there are differences in the frequency of certain signs between the uroarthritis and enteroarthritis groups. In patients with

Table 4 Interobserver reliability and intraobserver reproducibility with regard to radiological signs

\begin{tabular}{lllllll}
\hline & Interobserver A B & Interobserver A C & Interobserver B C & Intraobserver A & Intraobserver B & Intraobserver C \\
\hline $\begin{array}{l}\text { Syndesmophytes and } \\
\text { osteophytes (marginal/non- }\end{array}$ & 0.39 & 0.42 & 0.51 & 0.67 & 0.76 \\
$\begin{array}{l}\text { marginal/osteophyte) } \\
\text { Sacroiliitis (NY) grade 2-4 }\end{array}$ & 0.75 & 0.53 & 0.55 & 0.52 & 0.76 \\
Sacroiliitis (Stoke) grade 2-4 & 0.76 & 0.47 & 0.48 & 0.52 & 0.75 \\
Sacroiliitis (NY) grade 3-4 & 0.80 & 0.74 & 0.70 & 0.84 & 0.76 \\
Sacroiliitis (Stoke) grade 3-4 & 0.83 & 0.66 & 0.62 & 0.84 & 0.77 \\
liac enthesitis & 0.43 & 0.23 & 0.20 & 0.79 & 0.77 \\
Disc space narrowing & 0.53 & 0.38 & 0.59 & 0.64 & 0.69 & 0.68 \\
\hline
\end{tabular}

NY, New York criteria for sacroiliitis; Stoke, Stoke criteria for sacroiliitis. 
previous uroarthritis, syndesmophytes and sacroiliitis were found to be more common than in enteroarthritis. This difference reflects a worse prognosis and more frequent relapses of uroarthritis in this comparison and is in accordance with other studies. ${ }^{12} 131617$

Before the start of the study, we hypothesised that nonmarginal syndesmophytes would be more common in Chlamydia trachomatis triggered arthritis and in uroarthritis than in enteroarthritis. This was not confirmed, and our result suggests that the course of enteroarthritis and uroarthritis is very similar. In patients with syndesmophytes, the average number per patient and the average grade of syndesmophytes were also similar. Osteophytes were analysed in our study because it is sometimes hard to distinguish between syndesmophytes (especially non-marginal) and osteophytes. The distribution of the number and the frequency of occurrence were similar between the groups, suggesting that osteophytes did not introduce major bias into the study.

Assessment of agreement when interpreting enthesophytes (syndesmophytes and osteophytes) has not been common in earlier studies. In one study, interobserver and intraobserver agreement by radiologists in the presence of bridging syndesmophytes with regard to intervertebral spaces in the lumbar spine varied between 0.19 and $0.66 .{ }^{18}$ Their $\kappa$ values are not directly comparable with ours (interobserver agreement 0.39 to 0.51 and intraobserver agreement 0.67 to 0.76 ) because of differences in methods and definitions.

The grading of sacroiliac joints is considered difficult, and the $\kappa$ statistics to express intraobserver and interobserver variation have ranged from 0.07 to 1.0 and from 0.19 to 0.79 , respectively. ${ }^{19-23}$ In the analysis of this study, the original grading was divided dichotomously, with a cut off point between grades 1 and 2 or 2 and 3. This was done to enable us to take a majority decision with regard to the sacroiliac joint when there was a disagreement. Only independent blind readings were taken into account and reconciliation was not used, as it has been found to introduce an additional dimension to the variation and fails to provide a recipe for stable interpretations. ${ }^{21}$ In the determination of sacroiliitis a cut off point between grades 1 and 2 has provided good agreement $(\kappa=0.77$ and 0.765$)$ between radiologists when reading conventional radiographs. ${ }^{24-26}$ Intraobserver agreement has been reported to be good $(\kappa=0.765) .{ }^{25}$ In this study, the interobserver and intraobserver agreement in sacroiliitis grades 2-4 was from fair to good ( 0.53 to 0.75 and 0.52 to 0.75 , respectively). Analysis with a cut off point between grades 2 and 3 was also included because it has been considered pragmatically and statistically the best choice. ${ }^{21}$ In this comparison, interobserver and intraobserver agreement improved to the good to excellent range ( 0.70 to 0.80 and 0.77 to 0.84 , respectively). The use of the Stoke method of grading did not improve interobserver or intraobserver agreement, and with regard to reliability or reproducibility it cannot be seen to be superior to the New York grading system.

The association between grade 2-4 and grade 3-4 sacroiliitis (New York or Stoke) and the absence or lower incidence of disc space narrowing in the material was an unexpected finding. There was no significant difference in the average age of the patients, and age did not seem to be a source of bias. Sacroiliitis grade 2 or greater somehow seems to be protective against disc space narrowing. This relation may be associated with mechanical factors, such as the influence of inflammatory spinal disease on posture, as well as with genetic factors.

\section{Conclusions}

The lumbosacral radiographic features in patients with previous uroarthritis or enteroarthritis are characteristically similar, but in uroarthritis there are more syndesmophytes (both in overall incidence and in the numbers present) and more sacroiliitis. In the future, we anticipate that more sophisticated imaging methods (such as magnetic resonance imaging) will shed further light on this subject.

\section{Authors' affiliations}

L Kivisaari, L Laasonen, A Mannoja, J Pekkola, M Hämäläinen,

Department of Radiology, Helsinki University Central Hospital, Helsinki, Finland

M Leirisalo-Repo, Department of Medicine, Division of Rheumatology, Helsinki University Central Hospital

\section{REFERENCES}

1 Dougados M, van der Linden S, Juhlin R, Huitfeldt B, Amor B, Calin A, et al. The European Spondylarthropathy Study Group preliminary criteria for the classification of spondylarthropathy. Arthritis Rheum 1991;34:1218-27.

2 Aho K, Ahvonen P, Lassus A, Sievers K, Tiilikainen A. HLA B27 antigen and reactive arthritis. Lancet, 1973;ii, 157

3 Braun J, Gay K, van der Hejde D, Sieper J. On the difficulties of establishing consensus on the definition of and diagnostic investigations for reactive arthritis. Results and discussion of a questionnaire prepared for the 4 th International Workshop on Reactive arthritis, Berlin, Germany, July 3-6. J Rheumatol 1999:27:2185-92.

4 Pacheco-Tena C, Burgos-Vargas R, Vazquez-Mellado J, Cazarin J, PerezDiaz JA. A proposal for the classification of patients for clinical and experimental studies on reactive arthritis. J Rheumatol 1999;26:1338-46.

5 Inman RD. Classification criteria for reactive arthritis. J Rheumatol 1999;26:1219-21.

6 Gaston HJS, Lillicrap M. Arthritis associated with enteric infection. Best Pract Res Clin Rheumatol 2003;17:219-39.

7 Leirisalo-Repo M. Prognosis, course of disease, and treatment of the spondylarthropathies. Rheum Dis Clin North Am 1998:24:737-51.

8 McEwen C, DiTata D, Lingg C, Porini A, Good A, Rankin T. Ankylosing spondylitis and spondylitis accompanying ulcerative colitis, regional enteritis, psoriasis and Reiter's disease. Arthritis Rheum 1974;14:291-318.

9 Helliwell PS, Hickling V, Wright V. Do the radiological changes of classic ankylosing spondylitis differ from the changes found in the spondylitis associated with inflammatory bowel disease, psoriasis, and reactive arthritis? Ann Rheum Dis 1998;57:135-40.

10 Martel W, Braunstein EM, Borlaza G, Good AE, Griffin PE. Radiologic features of Reiter's disease. Radiology 1979;132:1-10.

11 Braun J. Imaging and scoring in ankylosing spondylitis. Best Pract Res Clin Rheumatol 2002;16:573-604

12 Good AE. Reiter's syndrome: long term follow-up in relation to development of ankylosing spondylitis. Ann Rheum Dis 1979;38(suppl 1):39-45.

13 Sairanen E, Paronen I, Mahonen H. Reiter's syndrome: a follow up study. Acta Med Scand 1969;185:57-63.

14 Van den Linden S, Valkenburg HA, Cats A. Evaluation of diagnostic criteria for ankylosing spondylitis. Arthritis Rheum 1984;27:361-8.

15 Dawes PT. Stoke ankylosing spondylitis spine score. J Rheumatol 1999:26:993-6.

16 Fox R, Calin A, Gerber RC, Gibson D. The chronicity of symptoms and disability in Reiter's syndrome. An analysis of 131 consecutive patients. Ann Intern Med 1979;91:190-3.

17 Leirisalo-Repo M, Helenius P, Hannu T, Lehtinen A, Kreula J, Taavitsainen M, et al. Long term prognosis of salmonella arthritis. Ann Rheum Dis 1997;56:516-20.

18 Vlam K, Mielants H, Veys EM. Involvement of the zygoapophyseal joint in ankylosing spondylitis: relation to the bridging syndesmophyte. J Rheumatol 1998;26:1738-45

19 Yazici H, Turunc M, Ozdogan H, Yurdakul S, Akinci A, Barnes CG. Observer variation in grading sacroiliac radiographs might be a cause of sacroiliitis reported in certain disease states. Ann Rheum Dis 1987;46:139-45.

20 Taylor HG, Wardle T, Beswick EJ, Dawes PT. The relationship of clinical and laboratory measurements to radiological change in ankylosing spondylitis. Br J Rheumatol 1991;30:330-5.

21 Hollingsworth P, Cheah P, Dawkins R, Owen E, Calin A, Wood P. Observer Variation in Grading Sacroiliac Radiographs in HLA-B27 Positive Individuals. J Rheumatol 1983;10:247-54.

22 van Tubergen A, Hofts-Dorenbosch L, Chulpen G, Landewe R, Wijers R, van der Heijde $D$, et al. Radiographic assessment of sacroiliitis by radiologists and rheumatologists: does training improve quality? Ann Rheum Dis 2003;62:519-25.

23 Spoorenberg A, de Vlam K, van der Heijde D, de Klerk E, Dougados $M$, Mielants $\mathrm{H}$, et al. Radiological scoring methods in ankylosing spondylitis: reliability and sensitivity to change over one year. J Rheumatol 1999;26:997-1002.

24 Blum U, Buitrago-Tellez C, Mundiger A, Krause T, Laubenberger J, Vaith P, et al. Magnetic resonance imaging (MRI) for detection of active sacroiliitis-a prospective study comparing conventional radiography, scintigraphy, and contrast enhanced MRI. J Rheumatol 1996;23:2107-14.

25 Docherty P, Mitchell MJ, MacMillan L, Mosher D, Barnes DC, Hanly JG. Magnetic resonance imaging in the detection of sacroiliitis. JRheumatol 1992;19:393-401.

26 Bellamy N, Newhook L, Rooney PJ, Brooks PM, Cockshott WP, Thompson GT, et al. Perception: a problem in the grading of sacro-iliac joint radiographs. Scand J Rheumatol 1984;13:113-20. 